\title{
Cerebral and orbital Aspergillus infection due to invasive aspergillosis of ethmoid sinus
}

\author{
J LOWE,* J BRADLEY, $\dagger$
}

*Department of Neuropathology, Queens Medical Centre, Nottingham, and the $†$ Department of Renal Medicine, Addenbrooks Hospital, Cambridge

SUMMARY Aspergillus fumigatus infection due to primary infection of the ethmoid sinus affected the brain and orbit of an otherwise healthy elderly woman. Primary nasal sinus infection may be $\mathrm{a}_{\dot{\omega}}$ chronic process analogous to aspergilloma of the lung: it may, however, assume a locally invasive ${ }_{-}$ course and spread to the orbit or brain, as in the case described. This form of Aspergillus infection is rare in Europe but common in hot dry areas like the Sudan. The infection was probably $A$ contracted by our patient while on holiday in South Africa.

Histological recognition of the condition is important in these unusual sites-many cases are $\vec{c}$ clinically diagnosed as a tumour and undergo biopsy.

\section{Case report}

A 74 year old woman collapsed while out shopping. On recovery she gave a history of intermittent frontal headaches, which she had had for one year since returning from a holiday to South Africa. Examination showed a right ptosis and proptosis, but full neurological examination was otherwise normal. She was admitted to hospital where she was noted to be intermittently confused and incontinent of urine.

Cranial radiographs and ultrasonographic examination of her right orbit yielded normal results. Computed tomography showed a large multilobular mass affecting the frontal poles, with peripheral enhancement and severe surrounding oedema (Fig. 1). There was also a low density mass obscuring the right ethmoid sinus, extending into the right orbit. This lesion was considered to be a primary cerebral tumour; surgery was not indicated. Her condition deteriorated, and she suffered increasing periods of confusion and drowsiness before her death.

Post mortem examination showed that the tumour was an abscess filled with cream coloured purulent material. It occupied the right frontal pole medially and extended into the left frontal lobe. The abscess was $4.5 \mathrm{~cm}$ in diameter and had irregular margins (Fig. 2). The inferior part of the cavity adhered to the orbital plate and surrounded the optic nerve close to its entry into the orbit. The bone was macroscopically intact. Examination of the right orbit showed the

Accepted for publication 11 February 1986 posterior structures to be obscured by firm white tissue with no spread to the globe. Similar firm white tissue without macroscopic necrosis obscured the ethmoid sinus on the right. The other nasal sinuses

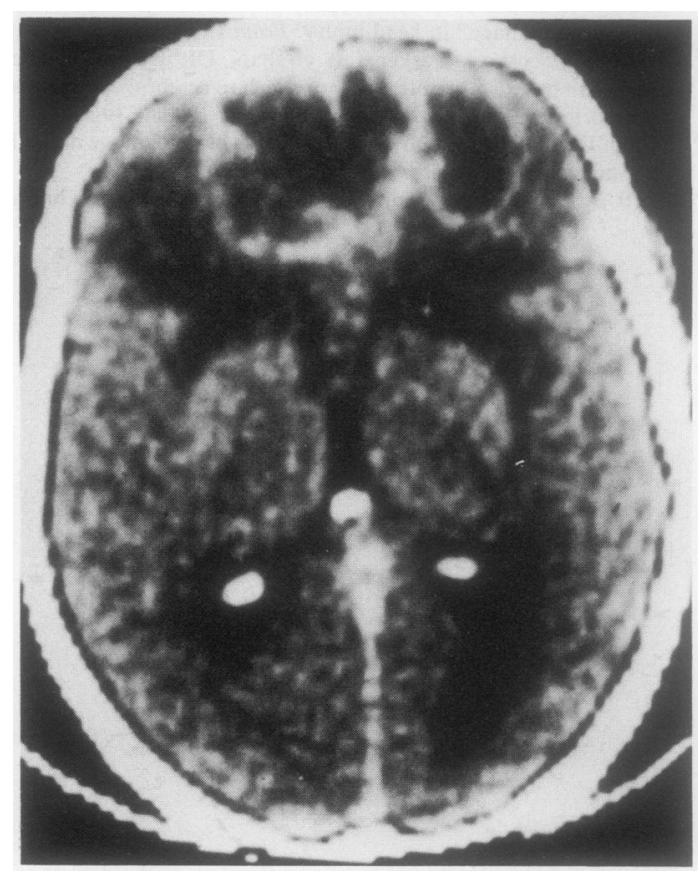

Fig. 1 Computed tomography scan of brain showing multilobular mass in frontal pole. 


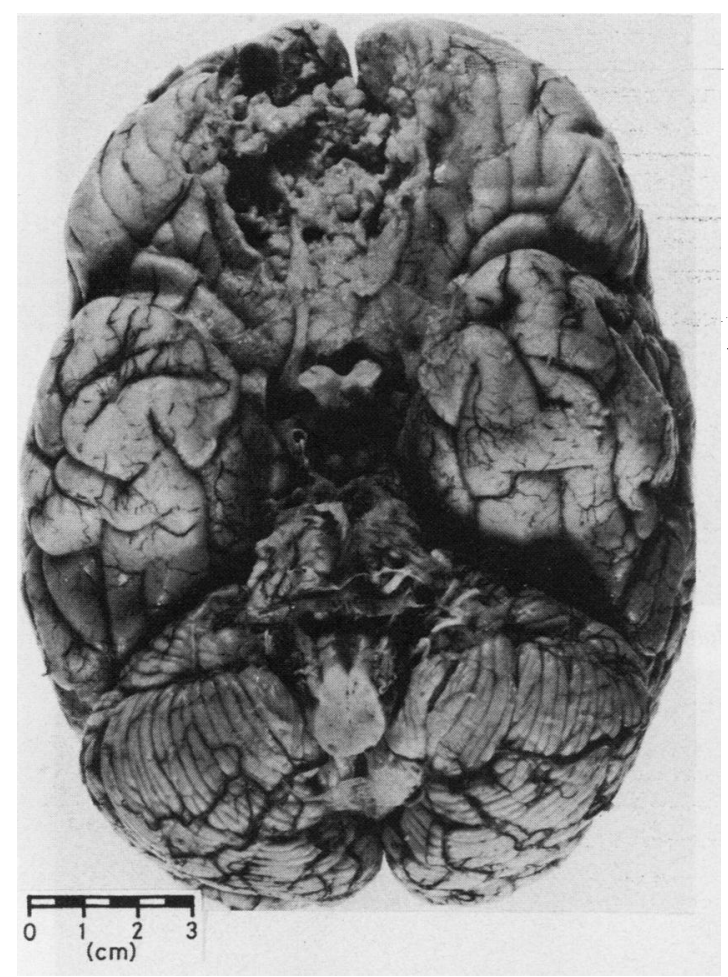

Fig. 2 Inferior aspect of brain showing frontal abscess.

were normal macroscopically. The other organs showed no macroscopic abnormalities.

Histology showed that the ethmoid sinus was obliterated by fibrous granulation tissue, with a heavy chronic inflammatory cell infiltrate. There were also discrete granulomata with histiocytic giant cells surrounding central zones of neutrophil leucocytes and fungal hyphae. Small granulomata were present in residual ethmoid sinus mucosa and also contained fungal hyphae (Fig. 3). Examination of the orbital contents showed a large serpiginous abscess cavity with surrounding dense fibrous tissue. This contained large numbers of partly degenerate fungal hyphae with a granulomatous reaction. The abscess did not affect the optic nerve (Fig. 4), or the globe. The cerebral abscess was surrounded by reactive astrocytes with a histiocytic and giant cell reaction. The cavity contained necrotic material, degenerate fungal hyphae, and neutrophil leucocytes (Fig. 5). No vascular invasion was seen in any of the tissues, but small arteries in the orbit, however, were occluded by old organising thrombus in relation to the margins of the abscess (Fig. 6). Histological examination of other tissues at necropsy showed no evidence of systemic infection or of any condition that may have predisposed the patient to an immune deficient state.

Culture of material from the cerebral abscess wall showed a pure growth of Aspergillus fumigatus.

\section{Discussion}

Spread to the nasal sinus by Aspergillus may take three forms, which parallel those seen in the lower respiratory tract; saprophytic infection (mycetoma), allergic aspergillosis, ${ }^{1}$ and invasive aspergillosis.

Saprophytic infection is a chronic indolent form of the disease localised to the nasal sinus. This is the most common form of sinus disease and is endemic in the Sudan. ${ }^{23}$ There are thought to be three predisposing factors to this high incidence in the Sudan: the dry climate, a high content of Aspergillus spores in the soil, and a high atmospheric dust content. Clinically, symptoms include headache, rhinorrhoea, and postnasal discharge.

Allergic Aspergillus sinusitis is a recently described condition in which the affected sinuses are filled with mucoid material containing numerous eosinophil leococytes, cellular debris, and Charcot-Leyden crystals. Scattered fungal hyphae are present. ${ }^{1}$

Invasive Aspergillus infection occurs when there is spread to the sinus wall and extension into the surrounding tissues. The reason for a saprophytic infection becoming invasive is not known. Invasive infection stemming from a pulmonary primary site is often seen in patients who are immunosuppressed. Notably, most of the published cases of primary rhinocerebral Aspergillus infection are not recorded as having a condition liable to cause an immunosuppressed state. The most common pathogenic organism in reported series of primary rhinocerebral aspergillosis has been Aspergillus flavus, but these cases are mostly from the Sudan and may reflect a local pattern of disease. Cases from outside the Sudan have shown other species including Aspergillus fumigatus. $^{2-478}$

Our patient probably developed a saprophytic sinus infection while she was on holiday in South Africa, producing a localised disease which resulted in the symptom of headache for one year. Invasive infection then developed with local extension into the orbit and brain.

In a review of thirty cases of orbital aspergillosis (the bulk of published cases) 21 had proptosis, 24 had nasal sinus disease, and six had cerebral disease. ${ }^{4}$ Initial diagnosis was made mainly after histological examination of biopsy material.

The main histological diagnostic feature of rhinocerebral aspergillosis is the presence of a histiocytic or giant cell reaction to fungal hyphae. The diagnosis of a fungal infection in this site should be considered whenever a biopsy, usually performed to make a 


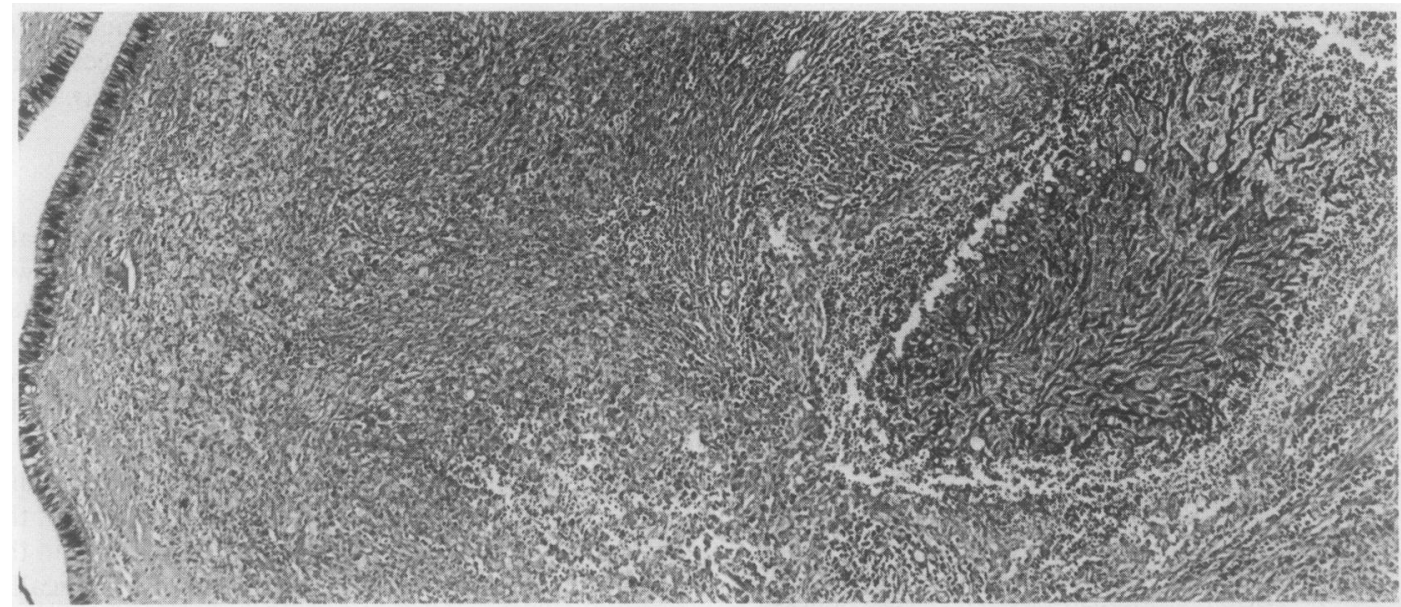

Fig. 3 Mucosa from ethmoid sinus showing Aspergillus granuloma (Periodic acid Schiff.) $\times 54$.

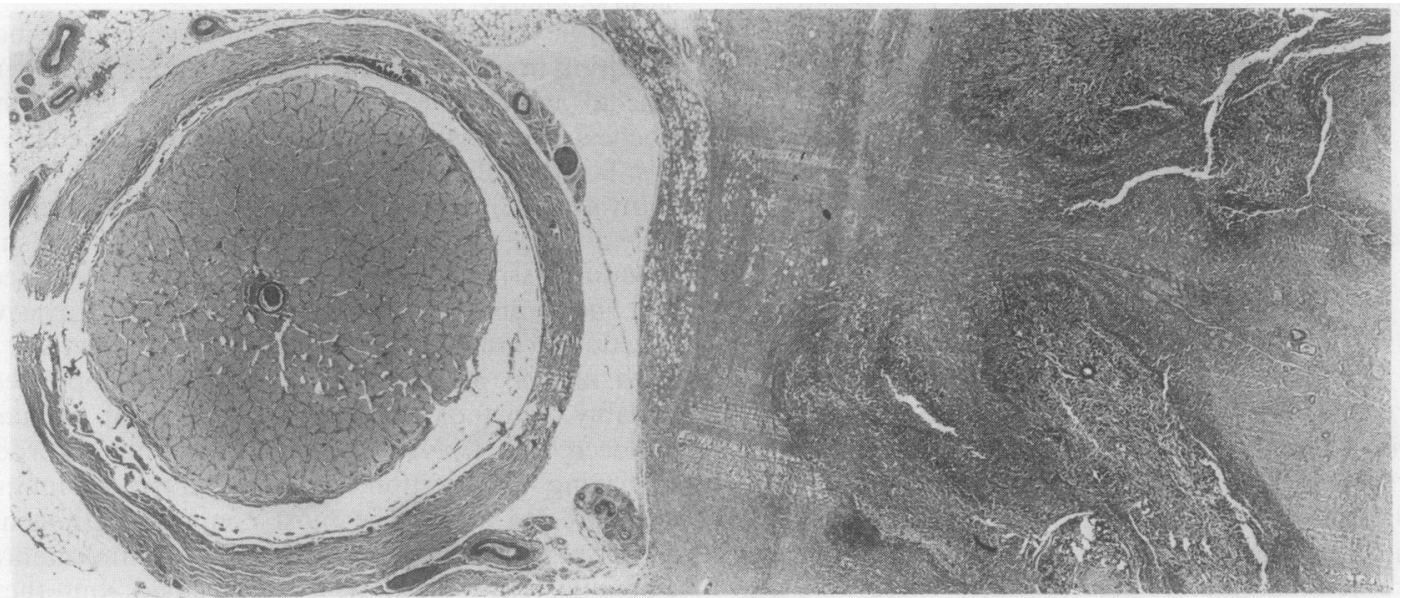

Fig. 4 Optic nerve (left) with margin of Aspergillus containing abscess. (Periodic acid Schiff.) $\times 13$.

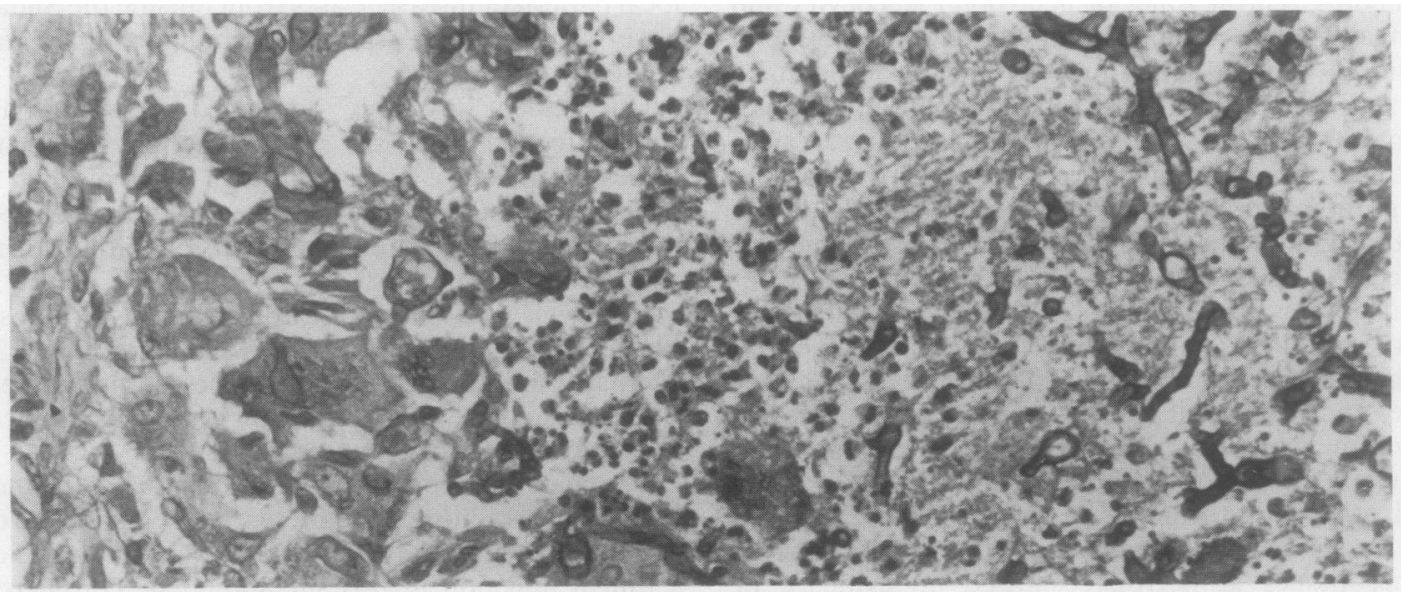

Fig. 5 Edge of cerebral abscess containing fungal elements. (Periodic acid Schiff.) $\times 340$. 


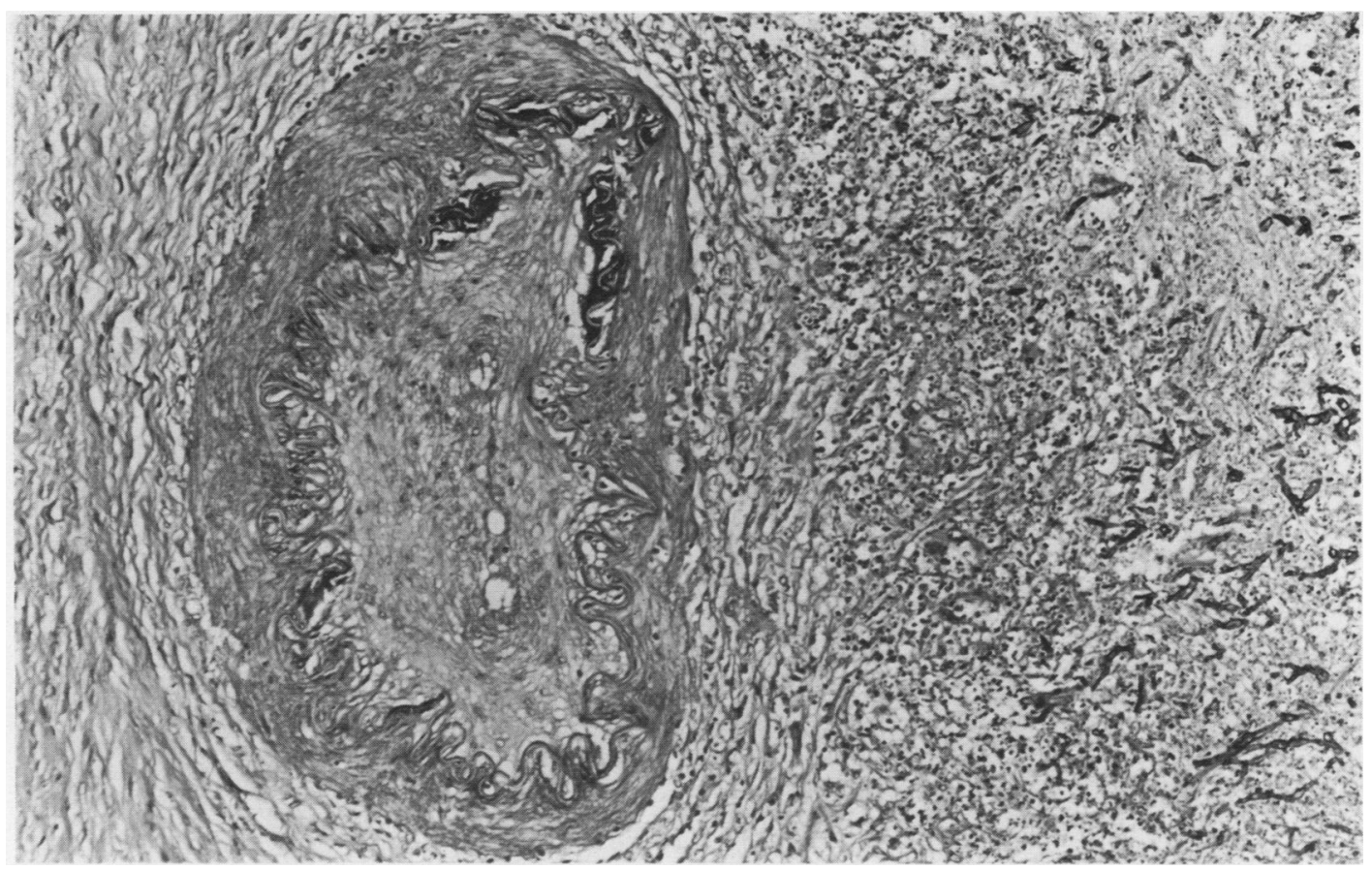

Fig. 6 Blood vessel from orbit at margin of abscess. (Periodic acid Schiff.) $\times 135$.

diagnosis of a tumour, contains necrotic tissue with occasional histiocytic giant cells. The identification of fungal hyphae in this type of necrotic tissue may be difficult due to degeneration and ballooning of the hyphae. The two main primary fungal infections affecting the region of the nasal sinuses and brain are Aspergillus and Zygomycosis (formerly phycomycosis). Histological sections show that the Aspergillus hyphae are thin $(3-6 \mu \mathrm{m})$, have regular parallel walls with easily seen septa, and show a regular dichotomous branching pattern at acute $45^{\circ}$ angles. In contrast, the hyphae in a zygomycotic infection are broad $(6-25 \mu \mathrm{m})$, have irregular contours to the hyphal walls, and have an irregular branching pattern with only very occasional septa. It is usually impossible, however, to identify a fungus in a small biopsy with a few degenerate hyphae without resorting to culture. Degenerate Aspergillus hyphae will lose septa and become swollen so that they resemble a zygomycosis. Care should therefore be exercised in the interpretation of biopsy material. ${ }^{56}$

A complication of cerebral invasion by aspergillosis is mycotic arteritis. This is due to direct invasion of small vessel walls by the fungal hyphae. In one reported case this led to fatal intracranial haemorrhage. ${ }^{7}$
Treatment of invasive rhinocerebral aspergillosis by antifungal chemotherapy has been regarded as being generally ineffective. Surgical excision is recommended as the most effective means of controlling the disease. $^{48}$

In conclusion, we have described a case of Aspergillus infection with a pattern, which, while well recognised, is very unusual in Europe. With the increase in travel to climates in which this pattern of disease is common, awareness of this type of presentation and biopsy diagnosis warrant wider consideration.

The culture of the Aspergillus was performed in the department of microbiology, Queens Medical Centre, Nottingham, by Janet Crowley. We are grateful to the Mycological Reference Laboratory, Colindale, for confirmation of the fungal type. We thank $\mathrm{Dr}$ PJ Toghill for permission to report this case. The photomicrographs were produced by $\mathrm{Mr}$ Bill Brackenbury of the department of histopathology.

\section{References}

${ }^{1}$ Katzenstein AA, Sale SR, Greenberger PA. Pathologic findings in allergic Aspergillus sinusitis. Am J Surg Pathol 1983;7:439-43. 
${ }^{2}$ Milosev B, Mahgoub S, Abdel Aal O, El Hassan AM. Primary aspergilloma of the paranasal sinuses in the Sudan. Br Surg 1969;56:132-7.

${ }^{3}$ Veress B, Malik OA, El Tayeb AA, El Daoud S, Mahgoub ES, El Hassan AM. Further observations on primary paranasal Aspergillus granuloma in the Sudan. Am J Trop Med Hyg 1973;22:765.

${ }^{4}$ Green WK, Font RL, Zimmerman LE. Aspergillosis of the orbit. Arch Ophthalmol 1969;82:302-13.

${ }^{5}$ Binford CH, Connor DH, eds. Pathology of tropical and extraordinary diseases. Vol. 2. Washington, DC: Armed Forces Institute of Pathology, 1976:564-5.

${ }^{6}$ Chandler FW, Kaplan W, Ajello L. A colour atlas and textbook of the histopathology of mycotic diseases. London: Wolfe Medical Publications, 1980:34-8, 122-7.

${ }^{7}$ Fernando SSE, Lauer CS. Aspergillus fumigatus infection of the optic nerve with mycotic arteritis of cerebral vessels. Histopathology 1982;6:227-34.

${ }^{8}$ Miglets AW, Saunders WH, Ayres L. Aspergillosis of the sphenoid sinuses. Arch Otolaryngol 1978;104:47-50.

Request for reprints to: Dr J Lowe, Department of Pathology, University Hospital, Queen's Medical Centre, Nottingham NG7 2UH, England. 